\title{
The Application of Image Recognition and Machine Learning to Capture Readings of Traditional Blood Pressure Devices: A Platform to Promote Population Health Management for Cardiovascular Diseases Prevention
}

\author{
Helen WY Lee ${ }^{1}$, Christopher TK Chu ${ }^{1}$, Karen KL Yiu ${ }^{2}$, Kelvin KF Tsoi ${ }^{1,2}$ \\ ${ }^{1} \mathrm{JC}$ School of Public Health and Primary Care \\ ${ }^{2} \mathrm{SH}$ Big Data Decision Analytics Research Centre \\ The Chinese University of Hong Kong \\ kelvintsoi@cuhk.edu.hk
}

\begin{abstract}
Digital solutions for Blood Pressure Monitoring (or Telemonitoring) have sprouted in recent years. Innovative solutions are often connected to the Internet of Things (IoT), mobile health (mHealth) platform for instance. However, clinical validity, technology cost and cross-platform data integration remain the major barriers to the application of these solutions. In this paper, we present an IoT-based and AI-embedded Blood Pressure Telemonitoring (BPT) system, which facilitates home blood pressure monitoring for individuals.

This system uses machine learning techniques to enable automatic digits recognition, with an F1 score of 98.5\%; and the cloud-based portal developed for automated data synchronization and risk stratification. Positive feedbacks on trial implementation are received from three clinics. The overall system architecture, development of machine learning model in digit identification, and cloud-based telemonitoring are addressed in this paper, alongside the followed implications.
\end{abstract}

\section{Introduction}

As an obstinate yet modifiable cardiovascular condition, hypertension is a major concern of many medical and public health practitioner. Medical groups, , such as European Society of Cardiology, European Society of Hypertension ${ }^{1}$, American Heart Society ${ }^{2}$, Australian expert group ${ }^{3}$, and Japanese Society of Hypertension, have well endorsed the clinical significance of out-of-office blood pressure, in both diagnosis and control. Out-of-office blood pressure measuring primarily refers to Ambulatory Blood Pressure Monitoring (ABPM) and Home Blood Pressure Monitoring (HBPM), where the latter refers to blood pressure (BP) self-management in home-setting on a day-to-day basis, usually in the morning and evening $^{3}$. Existing literature has found incremental risk for cardiovascular diseases in individuals with elevated home $\mathrm{BP}^{4,5,6,7,8,9}$ as well as strong association between morning BP readings given by HBPM and risk of stroke and coronary events. Crucially, HBPM's superior predictive values for cardiovascular events to office BP is equally highlighted ${ }^{10}$, owing to its validity and fidelity unaffected by white-coated effect and masked hypertension $^{11}$, while results from randomized controlled clinical trials have also favored HBPM in general BP outcomes.

BPT, as a form of out-of-office blood pressure monitoring has become an emerging trend in the last decade, gaining considerable recognition. It can render valuable health information and thus provide insightful guidance for antihypertensive medication prescription and dosage adjustment over clinical care ${ }^{13}$. It can also help facilitate early detection of hypertension and cardiovascular symptoms, as well as elevating health consciousness in the community.

In addition, telemedicine infrastructure is profoundly heightened for chronic diseases management in the midst of COVID-19 pandemic, during the times in which social distancing is much encouraged, and lockdown might even take place for sake of disease containment ${ }^{14}$. Reviews on impacts of telehealth have demonstrated similar health outcomes between remote monitoring and in-person care ${ }^{15}$. BPT, as facilitated by state-of-art technology, thereby serves as a safe, effective, and cost-effective alternative to inperson care in general.

In this paper, we proposed a development of a digital BPT solution of high compatibility with existing devices for BP capturing, storing and monitoring. This $\mathrm{BPT}$ solution includes image recognition of $\mathrm{BP}$ readings extracted from BP devices, cloud-computing techniques and web-portal for remote monitoring and related reminders or alert functions. 


\section{Background and Related Work}

BPT allows transmission of domestic BP readings remotely to healthcare facility ${ }^{12}$. Not only does BPT at home improve BP outcomes, such as reducing antihypertensive medication, but also minimizing therapeutic inertia, which is defined as unchanged treatment strategies despite deterioration in BP performance ${ }^{16}$.

Traditionally, hypertensive patients bring along their handwritten blood pressure records to clinic-visit for doctor's reference of their home BP profile. As a result, fragmented blood pressure records hampers the systematic collection and storage for building up individual health profile, one of the major limitations to diagnosis.

\subsection{Current BPT Technical Solutions}

With the advent of telemonitoring technology, a number of solutions have been invented. For example, automated upper-arm BP monitors (with internally installed data transmission function) such as Bluetooth BP monitors, were invented for easier data storage and transmission. Despite its high acceptability, accuracy and incremental benefits in long-term care including but not limited to better quality of life of patients ${ }^{12}$, amelioration on healthcare resources utilization and $\operatorname{costs}^{17}$ as well as, connectivity issue arises as another point of concern ${ }^{18}$. While its limited integration on application program interface (API) and service providers, narrow analytics ability such as data summary, coupled with its high technology-related costs, further offsets its cost-effectiveness. Given the cost reduction and popularity of smartphones as a result of plummeting costs of telecommunications, the mobile health market is surging correspondingly, with smartphones widely incorporated in BPT. Today, five billion of the world population owe mobile devices with over half of them are with smartphones ${ }^{19}$. Other solutions include (1) Smartphone applications coupled with external BP sensor or permitting manual BP input (function as BP e-journal), (2) smartphone-based cuffless BP monitors (such as finger photoplethysmography via smartphone camera), and (4) wearable devices, such as wrist tonometry-embedded smartwatches.

Despite enhanced affordability, doubts and concerns over these technologies remain. First and foremost, variations prevail in terms of the accuracy of BP monitors, resulting in invalid $\mathrm{BP}$ readings, which do little help or even lead to incorrect clinical decisions. According to the latest investigation by Picone on BP devices in marketplace ${ }^{20}$, only $8 \%$ of the wrist cuff devices and none of the cuffless wearable devices could pass the International Validation Standards. International medical groups such as British and Ireland
Hypertension Society (BIHS) ${ }^{21}$, also could not verify and recommend these alternative devices for measurements, like measuring pulse wave velocity, central blood pressure monitoring or arterial stiffness, given the absence of validation in the current stage. In other words, individual efforts made on HBPM could go in vain.

Besides, regarding the lack of API and service providers integration, limited analytical functions of these solutions have hampered their usage as clinical reference for healthcare professionals ${ }^{22}$, but merely for personal reference purpose. Hardly can HBPM and BPT interoperably exert their full potential in maximizing the clinical significance under such constraints and therefore worth of attention.

\subsection{Proposed BPT Digital Solutions}

Therefore, we proposed a novel BPT digital solution that overcomes the abovementioned barriers of existing solutions. Our system is made to aggravate clinically validated and accurate BP readings by smartphones' photo capturing function, from existing BP monitors that make use of seven-segment display screen, and communicate data among multiple platform, along with certain cloud-computing techniques for analytics upon data from BP logging.

The digits recognition focuses on seven-segment displays since it is of the highest adoptability among BP monitors, accounting for $97.2 \%$ of the recommended BP monitors for home-use, as advocated by BIHS ${ }^{21}$. A machine learning model is developed on the digit identification.

Cloud-server serves as a midpoint for data storage, integration and communication, and hence allowing real-time data synchronization and sharing for BPT, which exhibits in the form of web-portal system. On one hand, it provides analytics and reporting while on another hand, it triggers corresponding feedbacks, reminders and alert.

Our system strives to be a smart digital BPT solution of superb usability, cost-effective, validity, and intelligence, in replacement of traditional tedious handwritten $\mathrm{BP}$ recordings, exorbitant BPT technologies, unpromising devices. With the demonstration of our system, it is also hoped that similar application can be made on other important biometrics in managing chronic diseases, including blood glucose, weight and BMI, to name a few. Also, in contribution to the big data bank, guiding towards precision medicine and personalized health profile.

\section{System Architecture}

The proposed workflow home-based BPT system is illustrated in Figure 1. Health data capturing module for patients in form of mobile application platform, 
analytics cloud-server, web-portal module for healthcare professionals and an open RESTful API integration module are the core components for BPT.



Figure 1. The architecture of BPT digital solution

The mobile application allows an individual to capture BP readings on BP monitors by the smartphone camera, then the image is sent to the server via RESTful API-communication for the analysis of digits. The analysis of BP values is thus shown on the user's phone for confirmation. Upon submission, the $\mathrm{BP}$ values are sent to server for cloud storage. At the same time, healthcare workers in clinical settings could view the instantly updated profile via web portal. Follow-up actions can be executed when abnormal values are observed.

\subsection{Health Data Capturing Module - Image Recognition}

The two-step image capturing procedure involves taking an image with BP readings on BP monitors using the smartphone camera as the front-end, which are then transmitted to back-end analytics cloud server for further processing. For optimal image recognition at later stage, images are best taken at a horizonal level to BP monitors, appropriate distance between camera and the screen, and sufficient lighting so that the digits of BP readings are clearly visible with minimal reflection.

\subsubsection{Digit Location with a Pre-trained Neural Network}

An overview of the image recognition procedure is shown in Figure 2. The inputs of the digit location algorithm are primarily the images of BP monitors taken by smartphones as abovementioned. The images received in the server then undergoes an object detection process for digit location with a pre-trained neural network. Object detection locates all positions of digits of interest by bounding boxes as input and further labels and categories digits into corresponding groupings.

Images received are firstly automatically resized to $(446,416)$, they are then passed through a pretrained objection detection model for detection of digits location with number. By returning the coordinate of the bounding box of each digit, confidence level and digit number, digits with low confidence are eventually filtered out. All digits in the image are to be detected in this process.

\subsubsection{Clustering and Classification of Biometric Values.}

An algorithm was developed for grouping the detected digits in forming the physiological values of blood pressure. Clustering is performed on the bounding boxes coordinates for grouping detected digits into biometric readings with prior knowledge of three clusters in classifying systolic blood pressure (SBP), diastolic blood pressure (DBP) and heart rate (HR). Firstly, all bounding boxes coordinates are considered as potential exemplars, representative elements of clusters, and they are viewed as nodes in a network. Secondly, a number of real-valued messages is iteratively transmitted along the edges of the network so that a relevant set of exemplars and corresponding clusters is identified ${ }^{23}$

Let $\left\{S_{i j}\right\}, i=1, \ldots, N, j=1, \ldots, N$, be a set of $N^{2}$ where $S_{i j}$ denotes the similarity between two bounding boxes $x_{i}$ and $x_{j}$ in it. $S_{i j}$ is the negative of the square of their Euclidean distance; that is, $s(i, j)=-\left\|x_{i}-x_{j}\right\|^{2}$, $i \neq j$. The self-similarities $s_{k k}$ are referred to as "preferences" that affects the chance of one data point being an exemplar.

Two kinds of messages are made in the clustering and each takes into consideration a different kind of competition. One is named "responsibility" message as defined as $r(i, k)$, which is sent from data point $i$ to possible exemplar point $k$. $(i, k)$ represents how point $k$ is well suited to be the exemplar for point $i$, while taking other potential exemplars into consideration. The "responsibility" $(i, k)$ is updated as follows:

$$
r(i, k) \longleftarrow s(i, k)-\max _{k^{\prime} \text { s.t. } k^{\prime} \neq k}\left\{a\left(i, k^{\prime}\right)+s\left(i, k^{\prime}\right)\right\} .
$$

Another message named "availability" $(i, k)$ gather the evidence from data points as to see if each potential exemplar would make a good fit. It is sent from the representative point $k$ to point $i$, reflecting the accumulated evidence for how suitable it would be for point $i$ to choose point $k$ for its exemplar. Also, the evidence from other points that point $k$ should be an exemplar is taken into consideration. The "availability" $(i, k)$ is updated as follows: 


$$
a(i, k) \longleftarrow \min \left\{0, r(k, k)+\sum_{i^{\prime} \text { s.t. } i^{\prime} \notin\{i, k\}} \max \left\{0, r\left(i^{\prime}, k\right)\right\}\right\}
$$

After repetitive message passing, the criterion matrix $\mathrm{c}(i, k)$ can be formed by calculating the maximum of $a(i, k)+r(i, k)$ for point $i$.

$$
\mathrm{c}(i, k) \leftarrow \text { of } a(i, k)+r(i, k)
$$

The highest criterion value of each row of $\mathrm{c} c(i, k)$ is designated as the exemplar. Rows that share the same exemplar are in the same cluster ${ }^{24}$.

Three clusters ought to be formed upon identification and therefore remaining groups are filtered out (more than three clusters), and outlier clusters are filtered out. The remaining clusters are thus classified into meaningful biometric readings: SBP, DBP, HR, in accordance to the center coordinates of the cluster from top to bottom, left to right.

\subsection{Web-Portal Module}

This module provides a User Interface (UI) for healthcare providers to view the BP logging of their patients in our system. There are generated statistics about the BP patterns and highlights in different colors to be displayed in case of abnormality. Detailed health report sorting and searching can be done for quickly locating patients by personal identification numbers or BP ranges. A two-way API gateway can be set up between our analytics cloud server and external system to transmit information required including UUID, BP data, and report.

\subsection{Open RESTful API Module}

An open RESTful API module is designed to provide a handy protocol to integrate with other external systems. RESTful API design leveraging HTTP upon APIs usage ${ }^{25}$ is capable of fitting in nearly all existing protocols. Neither libraries nor extra software is required for REST API design. In this way, it permits integration with other applications along with our system, for example, Clinic Management System (CMS) in clinics and hereby provides more patient data for realtime analytics and timely monitoring.

\subsection{Neural Model for Image Recognition}

The most promising and validated methods for measuring BP are cuff-based and some wrist-based BP monitors with screen displays, despite the rise of multiple novel methods in blood pressure monitoring. The fundamental technologies of our system involve the BP capture empowered by digit recognition algorithms and the cloud-based server which facilitates data communication.

With application of our system, approximately all existing BP devices in the marketplace are catered with convenient data capture. The collected datacan later be summarised in a report and sent to affiliated healthcare providers regardless of the BP device used.



Figure 2. Overview of the image recognition procedure 


\section{Machine Learning Model for Automated Recognition of Biometric Readings}

A dataset was used in the development of machine learning algorithms. This dataset consisted of a total of 911 raw manually captured images of displays of 10 commonly used blood pressure monitors using seven segment digits, and were taken under realistic setting with smartphone camera to offer authentic dataset for training. The screen capture displays of BP monitors were contributed by a group of 50 healthy individuals with regular BP measurements.

\subsection{Raw Image of Blood Pressure Monitors}

The first dataset comprised of raw images of screen displays of blood pressure monitors under real scenario, by 50 healthy individuals using their own smartphones. Examples of images are displayed in Figure 2. The BP devices involved in the provision of raw images include Omron HEM-7200, A\&D UA853 while a variety of smartphone models were included in taking images, such as Samsung Galaxy S8, A9, and Note 8; iPhone 8, $\mathrm{X}$, and XS; Pixel, LG G4, and Huawei P10.

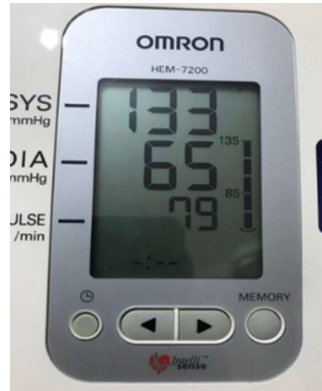

(a)

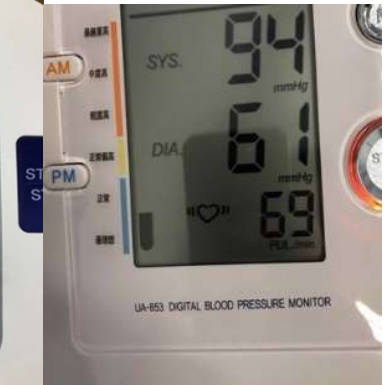

(b)
Figure 3. Examples of images taken of BP monitors for machine learning algorithms development (a) Omron HEM-7200 (b) A\&D UA853

Different environmental conditions are set up to mimic real-life situations: shooting angle, ambient light level, the distance between smartphone camera, and the screen display. Images were then randomly split into training and testing sets in ratio of $8: 2$, resulting 711 images in the training set and 200 images in the test set.

We made use of a free software, LabelImg, for annotating the graphical image of the dataset in deep learning. The digits in the images were then manually labelled independently by two investigators for validation while any discrepancies were solved upon consensus. Figure 4 shows some labelled images for later development of the algorithms.

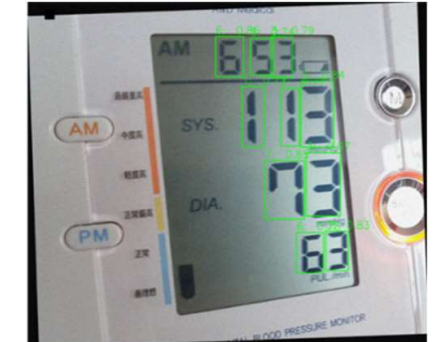

Figure 4. Example of labelled photos

\subsection{Synthetic Seven-segment Digits}

The second dataset consisted of auto-generated seven-segment digits. A synthetic dataset was spawned based on raw images for imitation, known as data augmentation, which aims at improving model accuracy, generalization, and controlling overfitting. We have yielded artificial data with techniques, with variations, in enhancing the volume and quality of training datasets thus establishing a better deep learning model $^{26}$. They are as follows.

- Geometric transformations: cropping, rotation, translation, noise introduction

- Color space transformations: modifications in greyscale intensity, brightness

Upon image manipulations, a total of 13,665 and 4760 images were included in the resulting dataset for training and testing.

\section{Development of Neural Network Model}

The final neural network model in recognizing seven-segment displays on BP monitors was built on a basis of object detection system, neural network architecture, alongside the techniques of transfer learning, augmentation and anchor box maximization. Details and steps in the development of the final model are explained below.

\subsection{Object Detection System}

You Only Look Once version 2 (YOLOv2) is adopted as the basic framework in object detection for building this neural network model. It offers real-time object detection with numerous categories. Substantial improvements in terms of speed and accuracy are observed in YOLOv2 compared with the previous model. Advantages include the ability to run at varying sizes of images, and novel training methods on object detection and classification including batch normalization, multi-scale training with input images of higher resolutions, utilizing higher spatial output in prediction of its final detection, and default bounding boxes rather than fully connected layers, of which 
elevated the performance of YOLO and thus the neural network model ${ }^{27}$. Despite vast improvements observed in accuracy in YOLOv2, it works below satisfaction upon small objects as the input down samplings would result in low dimension of feature map that supposedly used for final prediction. For better detection and analysis of BP digits, users of the application are educated with the technique of sizing BP readings as large as possible in the photos to ease the concern while using YOLOv2 in small objects.

\subsection{Neural Network Architecture}

ResNet-50 architecture is adopted here as the Residual Network applied on feature extraction, with prominent characteristic of adopting 'bottleneck' structure $^{28}$. Notwithstanding breakthroughs accomplished by neural networks in image classification, they are notorious for over-parametrized, redundant layers, as well as degradation issues ${ }^{29}$. Much of which are attributed to the reckless increase in network depth, with accuracy becomes saturated instead of constantly improved, and even sharp rise of training errors. Deep residual learning is thus intended to resolve these issues and optimize performance of neural network when it gets deeper ${ }^{27}$.

As suggested by its name, ResNet-50 is comprised of a classic residual block of 50 layers, per se having Convolutional (conv), Batch Normalization (Batch Norm), and Rectified Linear Unite (RELU) as elements in each layer. In ResNet-50, identity mapping is performed upon shortcut connections whereas the outputs are then added to those given by stacked layers. While it adopts Convolutional layers of 1 $\mathrm{x} 1,3 \times 3$ and $1 \times 1$, the 1 $\mathrm{x} 1$ layers are accountable for the reduction and restoration of dimension, which signify its 'bottleneck' structure. As a result, very deep networks are built by constructing and stacking residual blocks together. Figure 5 displays the workflow of ResNet-50 architecture.

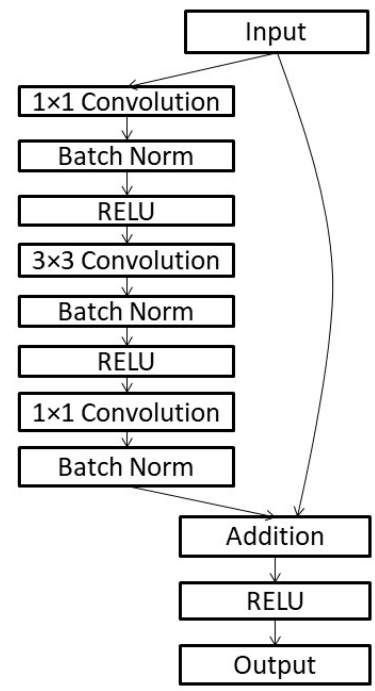

Figure 5. Display of ResNet-50 architecture

\subsection{Transfer Learning of SVHN Weights}

Transfer learning of SVHN weights serves as a major contributor in our machine learning algorithm for performance improvements. Transfer learning per se renders a seminal framework in improving performance of machine learning model without squandering efforts in valuable raw dataset labelling ${ }^{30}$. It is derived by the nature of features-specific of neural network trainings, enabling its capability in recognition among various datasets. First-layer features generally refer to the standard features found in the first layer, while last-layer features specific are those output units that are specific to a particular class, as trained by a supervised classification objective, in an N-dimensional SoftMax output layer.

In our model, pre-trained base network was developed with a base dataset, The Street View House Numbers (SVHN) dataset ${ }^{31}$, a dataset consisting of realworld images obtained from house numbers in Google Street View images, particularly leveraged in machine learning and object recognition algorithms development, with its signatures of minimal data pre-processing and formatting requirement. SVHN dataset is more preferable than MNIST in our training owing to its magnitude of labelled data (over 600,000 digit images), complexity of problems and high degree of simulation to reality. Through training of SVHN dataset, a weight was therefore obtained.

Afterwards, the pre-trained ConvNet with learned features was repurposed or transferred to the second target network for training the target dataset: raw dataset of BP monitors images and later synthetic dataset (see section 3.1), as a fixed feature extractor. The classified features were general and thus applicable to both base and our target datasets.

\section{Performance of the Developed Model}

The performance of the developed algorithms was evaluated by metrics of precision and recall. Precision is the proportion of actual digits identified correctly. Recall indicates the proportion of total relevant digits identified correctly out of all digits, and that indicates the model's ability to identify digits of interest in a dataset. The overall accuracy is measured by F1 score which takes both precision and recall into considerations.

$$
\begin{aligned}
\text { Precision } & =\frac{\text { True Positive }}{\text { True Positive }+ \text { False Positive }} \\
\text { Recall } & =\frac{\text { True Positive }}{\text { True Positive }+ \text { False Negative }} \\
\text { F1 } & =2 \times \frac{\text { Precision } \times \text { Recall }}{\text { Precision }+ \text { Recall }}
\end{aligned}
$$


A total of six experimental steps were executed in a bid to produce a model of optimal performance. The final model in digit recognition attained precision of $98.5 \%$, recall of $98.4 \%$ and $\mathrm{F} 1$ score of $98.5 \%$. Details are displayed in Table 1.

\subsection{Training from Scratch}

As anticipated, training from scratch with merely raw images gave very low accuracy, as demonstrated by all performance metrics (precision, recall and F1 score). The core of the problem is the small dataset size, 911 images are far from sufficient to train a deep learning model.

\subsection{Introduction of Transfer Learning of SVHN Weights}

Detailed illustration concerning methodology of transfer learning of SVHN weights is described in section 4.3. With the entry of transfer learning of SVHN weights and training starting from the $10^{\text {th }}$ layer, drastic improvements were observed in model performance. Metrics instantly increased precision from $7.1 \%$ to $89.7 \%$, recall from $2.7 \%$ to $72.6 \%$, and $\mathrm{F} 1$ score from $3.9 \%$ to $80.3 \%$.

The main reason is that the SVHN dataset and our dataset are similar by nature as both are predicting digits from 0-9. The first few features extracting layers of the network are mainly to extract general features on the digits such as shape and the outline. As SVHN digits and seven-segment digits are similar in terms of general features, freezing one fifth of the layers during training can successfully transfer the general shape features in SVHN shape in predicting seven-segment digits while retaining 4/5 layers for extracting seven-segment digits specific features.

\subsection{Image Enhancement with Adaptative Histogram Equalization (AHE)}

Adaptative histogram equalization (AHE) $)^{32}$ was performed with an aim to remove unfavorable illumination effects in the images and optimizing contrast. However, only minimal elevation achieved in terms of precision, but significant degradations in F1 and recall were noticed, compared to step 2. Therefore, AHE was abandoned in developing the model.

\subsection{Augmentation}

Augmentation to the original images with around 7 times each gave rise to images of around 5000 and 1400 in training and testing sets respectively. We randomly applied 2 to 3 augmentation techniques to each image include flipping on horizontal or vertical axis, rotating an image with certain degree, randomly cropping a section from given image, and adding Gaussian noise and random colour manipulation. Compared with step 2, introduction of augmentation with training of synthetic dataset gave rise to certain improvements in the model, precision from $89.7 \%$ to $90.7 \%$, recall from $72.6 \%$ to $77.0 \%$ and $\mathrm{F} 1$ score from $80.3 \%$ to $85.1 \%$. Thereby, augmentation is adopted as a better tool in model development than AHE.

Continuous refinements observed upon further application of augmentation techniques at around 14 times. However, model performance started to be saturated and stagnate despite ongoing augmentation.

\subsection{Anchor Box Maximization}

In a bid to mitigate the issues about stagnation of model performance upon abundance augmentation, anchor box maximization technique is adopted in the final model (marked with asterisk* in the table). Owing to the inherent deficiency of YOLOv2 in recognition small object, cropping original images to maximization each anchor box was sought to be a resolution in upgrading the performance of the final model. The illustration is shown in Figure 6. Prompt breakthrough was achieved, contributing to over $98 \%$ in all three performance metrics.

Maximization of anchor box size in each image is achieved by cropping all irrelevant space to maximize the relative size of anchor box size in each image. Demonstration of before and after anchor box maximization is shown below in Figure 6.

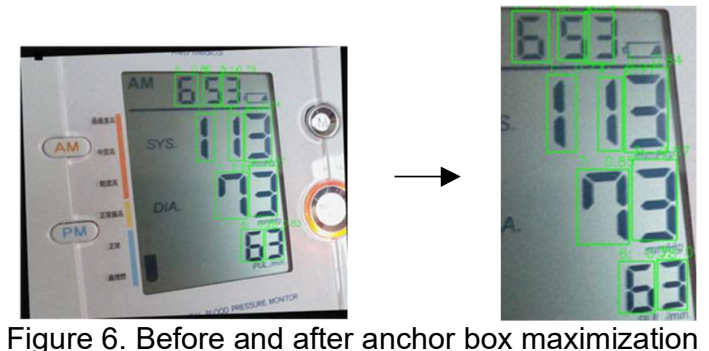

6.6. Real-life Implementation Performance. Aside from the experimental setting, performance of the model is also tested in a real-life setting for pilot trial and performance is calculated in accuracy.

$$
\text { Accuracy }=\frac{\text { Number of correctly classified images }}{\text { Total number of images }}
$$

Our mobile app of HealthCap has been released in iOS and android since May 2019 in Hong Kong. It is free to download for people with health concerns to record their BP with aforementioned pre-training model embedded. With data of 4766 images from 348 users in real-life setting, the overall accuracy in digits recognition of (1) all three digits, (2) SBP only, (3) DBP only, (4) HR only, are $82.2 \%, 91.7 \%, 91.2 \%$ and $87.7 \%$ respectively. The accuracy is comparable to our 


\begin{tabular}{|c|c|c|c|c|c|}
\hline & Training Description & Number of images & Precision & Recall & F1 Score \\
\hline 1 & $\begin{array}{l}\cdot \text { Raw images } \\
\cdot \text { Training from scratch }\end{array}$ & $\begin{array}{l}\text { Train: } 711 \\
\text { Test: } 200\end{array}$ & $7.1 \%$ & $2.7 \%$ & $3.9 \%$ \\
\hline 2 & $\begin{array}{l}\text { - Transfer learning of SVHN weights } \\
\text { - Starting from the } 10^{\text {th }} \text { layer }\end{array}$ & $\begin{array}{l}\text { Train: } 711 \\
\text { Test: } 200\end{array}$ & $89.7 \%$ & $72.6 \%$ & $80.3 \%$ \\
\hline 3 & $\begin{array}{l}\text { - Transfer learning of SVHN weights } \\
\text { - Starting from the } 10^{\text {th }} \text { layer } \\
\text { - Global contrast optimized in all images using adaptive } \\
\text { histogram equalization }\end{array}$ & $\begin{array}{l}\text { Train: } 711 \\
\text { Test: } 200\end{array}$ & $90.2 \%$ & $62.9 \%$ & $74.1 \%$ \\
\hline 4 & $\begin{array}{l}\text { - Transfer learning of SVHN weights } \\
\text { - Starting from the } 10^{\text {th }} \text { layer } \\
\text { - Image Augmentation to produce } 7 \text { times more images }\end{array}$ & $\begin{array}{l}\text { Train: } \sim 5000 \\
\text { Test: } \sim 1400\end{array}$ & $90.7 \%$ & $77.0 \%$ & $83.4 \%$ \\
\hline 5 & $\begin{array}{l}\text { - Transfer learning of SVHN weights } \\
\text { - Starting from the } 10^{\text {th }} \text { layer } \\
\text { - Image Augmentation to produce } 14 \text { times more images }\end{array}$ & $\begin{array}{l}\text { Train: } 10665 \\
\text { Test: } 3000\end{array}$ & $94.0 \%$ & $77.8 \%$ & $85.1 \%$ \\
\hline 6 & $\begin{array}{l}\text { - Transfer learning of SVHN weights } \\
\text { - Starting from the } 10^{\text {th }} \text { layer } \\
\text { - Crop original images to maximise each anchor box* } \\
\text { - Augmentation to produce } 20 \text { times more images }\end{array}$ & $\begin{array}{l}\text { Train: } 13665 \\
\text { Test: } 4760\end{array}$ & $98.5 \%$ & $98.4 \%$ & $98.5 \%$ \\
\hline
\end{tabular}

experimental results, providing strong evidence for its applicability to BP devices in the market. Besides, this model can be adopted in catering blood glucose readings recording with promising accuracy.

\section{Blood Pressure Telemonitoring: Cloud-web Portal}

Process automation has to be well-embedded in the BPT platform, from initial data synchronization to risk classification and to health report generation, in striving for efficient telemonitoring and timely care.

\subsection{Data Synchronization}

Prompt data synchronization is a prerequisite for telemonitoring and therefore the proposed application is able to deliver real-time data fetch from cloud sever to another side, example receivers can be healthcare providers or caregivers, to name a few. Prototype of cloud-web portal is shown in Figure 1. Multiple access to data and individual's profile is also supported. For example, access is granted to different clinics upon users' consent.

\subsection{Automated BP Risk Level Classification}

Cloud computing technics are exerted in classifying individual BP risk level based on their BP records, for heightening efficiency in managing a host of individuals at the same time. With reference to 2020 guidelines given by Hypertension Canada ${ }^{33}, 135 / 85 \mathrm{mmHg}$ observed in Home BP series is used as the threshold for hypertension diagnosis. Therefore, BP readings with over $135 / 85 \mathrm{mmHg}$ are regarded as 'hypertensive readings' and used to as a parameter in the computing algorithm

for detecting BP risk levels, which can be high, medium, and low. Criteria for classification are as follows.

- Relax Group (low risk): $\leq 20 \%$ are hypertensive SBP readings

- $\quad$ Leisure Group (medium risk): $>20 \% \& \leq$ $50 \%$ are hypertensive SBP readings

- Caring group (high risk): $>50 \%$ are hypertensive SBP readings

Such classification is of clinical significance in formulating personalized care plan for individuals, especially for individuals of caring group, whose BP are not well-controlled. Meanwhile, for individuals whose $\mathrm{BP}$ are well-controlled (stable cases) observed via remote monitoring, it is feasible for them to reduce the number of clinical visits, which in turns help reduce the burden of health system.

With sufficient BP data accumulated over time, detailed personal BP record and profile can be built and these data are of paramount importance in calculating relevant indicators regarding Blood Pressure Variability and Cardiovascular risk (Q-risk), also with automation upon computing techniques in the system. Hence, providing healthcare workers with more clinical clues in handling the condition of each patient. 


\subsection{Automated Health Report Generation}

Individual BP health can be scrutinized with the help of automatically generated health report in PDF documents, which is of easy printing and reading, thus facilitating healthcare workers in explaining one's conditions to individuals and issuing corresponding follow-up actions.

\subsection{Overall Feedbacks}

A total of three clinics have adopted this BPT system for trials, most of which responded positively to the overall solution, particularly in terms of usability and validation. Data storage and management are much aided with the BPT-portal system while facilitates close monitoring on individuals' health frequent in-person care.

Thus, remote monitoring is tested to be feasible with the proposed BTP tool, especially during times of infectious disease outbreak (covid-19).

\section{Conclusion and Future Work}

A promising digital solution, HealthCap, using for $\mathrm{BPT}$ is presented in this paper, with implementation on smartphones and cloud-server. Important elements of this BPT digital solution are the datasets, both raw and synthetic; neural network model development, where multiple methods are involved in refining the accuracy of the digits recognition of seven-segment displays; and cloud-web portal platform, that leads to easy telemonitoring effects.

The digit recognition algorithm attained F1 score of $98.5 \%$ in experimental setting while accuracy of around $91 \%$ in real-life setting. Despite slight drop in real-life setting, overall performance of the proposed model is satisfactory in both experimental and real-life settings. On the other hand, the application of cloud-web server is smooth in real-life setting, with positive feedbacks from healthcare workers in collaborating clinics.

The interoperability between mHealth platform embedded with machine learning algorithms for digits recognition and cloud-driven web portal eases concerns regarding clinical validity and cost. Thus, it serves as a resort which surmounts the limitations of current available BPT digital solutions.

\section{References}

[1] G. Parati, G.S. Stergiou, R. Asmar, G. Bilo, P. de Leeuw, Y. Imai , K. Kario, E. Lurbe, A. Manolis, T. Mengden, E. O'Brien, T. Ohkubo, P. Padfield, P. Palatini, T. Pickering, J. Redon, M. Revera, L.M. Ruilope, A. Shennan, J.A. Staessen, A. Tisler, B. Waeber, A. Zanchetti, G. Mancia; ESH Working Group on Blood Pressure Monitoring. European Society of Hypertension guidelines for blood pressure monitoring at home: a summary report of the Second International Consensus Conference on Home Blood Pressure Monitoring. J Hypertens. 2008;26:15051526.

[2] American College of Cardiology. 2017 Guideline for the Prevention, Detection, Evaluation, and Management of High Blood Pressure in Adults; 2017 [cited 25 January 2019].

[3] J. Sharman, F. Howes, G. Head, B. McGrath, M. Stowasser, M. Schlaich et al. Home blood pressure monitoring: Australian Expert Consensus Statement. Journal of Hypertension. 2015;33(9):1721-1728.

[4] T. Ohkubo, Y. Imai, I. Tsuji, K. Nagai, J. Kato, N. Kikuchi, A. Nishiyama, A. Aihara, M. Sekino, M. Kikuya, S. Ito, H. Satoh, S. Hisamichi. Home blood pressure measurement has a stronger predictive power for mortality than does screening blood pressure measurement: a populationbased observation in Ohasama, Japan. J Hypertens. 1998;16:971-975.

[5] R. Sega, R. Facchetti, M. Bombelli, G. Cesana, G. Corrao, G. Grassi, G. Mancia. Prognostic value of ambulatory and home blood pressures compared with office blood pressure in the general population: follow-up results from the Pressioni Arteriose Monitorate e Loro Associazioni (PAMELA) study. Circulation. 2005;111:1777-1783. doi: 10.1161/01.CIR.0000160923. 04524.5B

[6] M.R. Hänninen, T.J. Niiranen, P.J. Puukka, J. Johansson, A.M. Jula. Prognostic significance of masked and whitecoat hypertension in the general population: the FinnHome Study. J Hypertens. 2012;30:705-712. doi: 10.1097/HJH.0b013e328350a69b

[7] D. Tientcheu, C. Ayers, S.R. Das, D.K. McGuire, de Lemos JA, Khera A, Kaplan N, Victor R, Vongpatanasin W. Target organ complications and cardiovascular events associated with masked hypertension and whitecoat hypertension: analysis From the Dallas Heart Study. J Am Coll Cardiol. 2015;66:2159-2169. doi: 10.1016/j.jacc.2015.09.007

[8] A. Ntineri, P.G. Kalogeropoulos, K.G. Kyriakoulis, E.K. Aissopou, G. Thomopoulou, A. Kollias, G.S. Stergiou. Prognostic value of average home blood pressure and variability: 19-year follow-up of the Didima study. J Hypertens. 2018;36:69-76. doi: 10.1097/HJH.0000000000001497

[9] K. Okumiya, K. Matsubayashi, T. Wada, M. Fujisawa, Y. Osaki, Y. Doi, N. Yasuda, T. Ozawa. A U-shaped association between home systolic blood pressure and four-year mortality in community-dwelling older men. J Am Geriatr Soc. 1999;47:1415-1421.

[10] S. Fuchs, R. de Mello, F. Fuchs. Home Blood Pressure Monitoring Is Better Predictor of Cardiovascular Disease and Target Organ Damage than Office Blood Pressure: A 
Systematic Review and Meta-Analysis. Current Cardiology Reports. 2013;15(11).

[11] B. Cheung, K. Ong, R. Leung, L. Wong, Y. Song, P. Sham. Single-nucleotide polymorphisms near the microsatellite D17S1303 and the development of hypertension in a 6-year longitudinal study. Journal of Human Hypertension. 2007;22(2):151-153.

[12] S. Omboni, R. Ferrari. The Role of Telemedicine in Hypertension Management: Focus on Blood Pressure Telemonitoring. Current Hypertension Reports. 2015;17(4)

[13] K. Kario, D. Shimbo, S. Hoshide, J. Wang, K. Asayama, T. Ohkubo et al. Emergence of Home Blood PressureGuided Management of Hypertension Based on Global Evidence. Hypertension. 2019;74(2):229-236.

[14] J. Portnoy, M. Waller, T. Elliott. Telemedicine in the Era of COVID-19. The Journal of Allergy and Clinical Immunology: In Practice. 2020;8(5):1489-1491.

[15] G. Flodgren, A. Rachas, A. Farmer, M. Izitari, S. Shepperd. Interactive telemedicine: effects on professional practice and health care outcomes. Cochrane Database of Systematic Reviews. 2015;

[16] R. Agarwal, J. Bills, T. Hecht, R. Light. Role of Home Blood Pressure Monitoring in Overcoming Therapeutic Inertia and Improving Hypertension Control. Hypertension. 2011;57(1):29-38.

[17] S. Omboni, T. Gazzola, G. Carabelli, G. Parati. Clinical usefulness and cost effectiveness of home blood pressure telemonitoring. Journal of Hypertension. 2013;31(3):455-468

[18] E. Finnegan, M. Villarroel, C. Velardo, L. Tarassenko. Automated method for detecting and reading sevensegment digits from images of blood glucose metres and blood pressure monitors. Journal of Medical Engineering \& Technology. 2019;43(6):341-355.

[19] L. Silver. Smartphone Ownership Is Growing Rapidly Around the World, but Not Always Equally [Internet]. Pew Research Center. 2020 [cited 30 June 2020]. Available from: https://www.pewresearch.org/global/2019/02/05/smartp hone-ownership-is-growing-rapidly-around-the-worldbut-not-always-equally/

[20] D. Picone, R. Deshpande, M. Schultz, R. Fonseca, N. Campbell, C. Delles et al. Nonvalidated Home Blood Pressure Devices Dominate the Online Marketplace in Australia. Hypertension. 2020;75(6):1593-1599.

[21] British and Irish Hypertension Society. How to Use the List of Validated Monitors [Internet]. 2020 [cited 30 June 2020]. Available from: https://bihsoc.org/bp-monitors/
[22] S. Omboni, E. Panzeri, L. Campolo. E-Health in Hypertension Management: an Insight into the Current and Future Role of Blood Pressure Telemonitoring. Current Hypertension Reports. 2020;22(6).

[23] B. J. Frey, D. Dueck, "Clustering by passing messages between data points," Science, vol. 315 , no. 5814, pp. 972-976, 2007.

[24] K. Zhang, X. Gu. 2020. An Affinity Propagation Clustering Algorithm For Mixed Numeric And Categorical Datasets

[25] R. Fielding. Architectural styles and the design of network-based software architectures. University of California, Irvine; 2000.

[26] C. Shorten, T. Khoshgoftaar. A survey on Image Data Augmentation for Deep Learning. Journal of Big Data. 2019;6(1)

[27] J. Redmon, A. Farhadi. YOLO9000: Better, Faster, Stronger. 2017 IEEE Conference on Computer Vision and Pattern Recognition (CVPR) [Internet]. 2017 [cited 6 July 2020]. p. 6517-6525. Available from: https://ieeexplore.ieee.org/document/8100173

[28] K. He, X. Zhang, S. Ren, J. Sun. Deep residual learning for image recognition. In: Proceedings of the IEEE Conference on Computer Vision and Pattern Recognition. 2016:770-778.

[29] G. Tzelepis, A. Asif, S. Baci, S. Cavdar, E. Aksoy . Deep Neural Network Compression for Image Classification and Object Detection. 2019 18th IEEE International Conference On Machine Learning And Applications (ICMLA) [Internet]. IEEE; 2019 [cited 7 July 2020]. Available https://ieeexplore.ieee.org/document/8999138/

[30] S. Pan, Q. Yang. A Survey on Transfer Learning. IEEE Transactions on Knowledge and Data Engineering. 2010;22(10):1345-1359.

[31] Y. Netzer, T. Wang, A. Coates, A. Bissacco, B. Wu, and A.Y. Ng. Reading digits in natural images with unsupervised feature learning. Deep Learning and Unsupervised Feature Learning Workshop, NIPS; 2011.

[32] D. Sonker, M.P. Parsai. Comparison of histogram equalization techniques for image enhancement of grayscale images of dawn and dusk. Int J Mod Eng Res. 2013;3(4):2476-2480. Available from: www.ijmer.com.

[33] D. Rabi, K. McBrien, R. Sapir-Pichhadze, M. Nakhla, S. Ahmed, S. Dumanski et al. Hypertension Canada's 2020 Comprehensive Guidelines for the Prevention, Diagnosis, Risk Assessment, and Treatment of Hypertension in Adults and Children. Canadian Journal of Cardiology. 2020;36(5):596-624. 